\title{
271
}

sie einen grösseren Umfang erreichten oder nach innen in das Darmlumen prominirten; obgleich ihr Vorkommen ausserordentlich selten zu sein scheint, so kann man doch in Zukunft bei der Diagnostik rundlicher, beweglicher, durch die Bauchdecken fühlbarer Knoten auf solche Fibroide Rücksicht nehmen.

Uebrigens wird es wohl nothwendig werden, im System der Geschwälste, derartige fast rein aus glatten Muskelfasern bestehende Geschwülste, wie sie auch im Uterus nicht seiten vorkommen und jeb auch in der Haut beobachtet babe, von denen, welche rein aus Bindegewebe bestehen, zu trennen, wenn auch die Praxis beide Formen fortwährend zusammenhalten wird. Man könnte vielleicht den Namen Fibroid für beide Formen beibehalten, die reinen Bindegewebsgeschwülste aber mit Verneuil: Fibrome nennen und die reinen Muskelfasergesehwülste; Myome. Der letztere Name ist freilich schon für die aus quergestreiften Muskeln hestebenden Neubilduagen vorgeschlagen*), aber da letztere ganz ausserordentlich seiten vorkommen und nie ganz rein selbstständige Tumoren bilden, bedïrfen sie kaum einen besonderen Gattungsnamen.

4.

\section{Ein Fall von Markschwamm mit ungewöhulich vielfacher metastatischer Verbreitung.}

\author{
Von Prof, Förster in Göttingen.
}

Der Kranke (Heinricl Becker, 37 Jalure alt, aus Wiershaussen) war früher stets gesund und bemerkte zuerst in Januar 1857, dass sich um das linke Huiftbein cinzelne harte Anschwellungen bildeten; dieselben wurden bald grösser und behinderten ibn am Gehen. Im April bildeten sich einige Knoten an der linken und später auch der rechten Submaxillargegend; Anfang Mai entwickelten sich auf dem behaarten Kopfe einige Knoten, dann zeigten sich solche in den Achselhöhleu und in der Haut der Brust, zuletzt auch in dev Schilddrüse. Der Kranke magerte dabei sehr ab, klagte besonders über heftigen Sclumerz im Epigastrium, wo starke Pulsationen der Aorta fühlbar waren, endlich traten profuse Durchfälle ein und am 3. Juli erfolgte der Tod. Seine geistigen Functionen hlieben bis zum Tode ungestört.

Die am folgenden Tage vorgenommene Section ergab: Die Leiche zeigte einen hohen Grad von Abmagerung und Blutarmutl, die Haut war blass, reinlich weiss. In der behaarten Haut des Kopfes sassen 6 runde Krebsknoten von 6-12 Lin. Durchmesser, dieselben gingen vom subcutanen Zellgewebe aus, prominirten nach aussen, die sie bedeckende Haut nässte bei den meisten. In der Haut der Brust und des Bauches sizen eine Anzal kleinere rundliche und flache Krebsknoten,

*) Diess ist wohl ein Irrthum; ich hube den Namen gerade für Geschwilste aus glatten Muskeln proponirt (Archiv VI. S. 553), wabrend ich die aus quergestreiften Muskeln bestehenden Myosarcome nannte (Würzb. Verb. I. S. 191).

Virchow. 
die kleinsten sind $3-4$ Lin. breit, $\frac{1}{2} \cdots 1$ Lin. dick; alle geben vam submucösen Zellgewebe aus.

Die Schilddriise ist vergrössert und hat die Form einer lobulären Struma, am meisten ist das linke Horn vergrössert. Die Schnittfläche zeigt dicht aneinander gedrăngte, vielfach confluirende, rundliche Krehsknoten, während das Drüsengewebe bis auf geringe Spuren zwischen den Knoten völlig verschwunden ist, so dass das, was man unter der Form der Schilddrüse vor sich hat, eigentlich nichts als eine an deren Stelle getretene Markschwammmasse darstellt. (Es ist dies der erste Fall von secundärer Krebsbildung in der Schilddrüse, den ich beobachtete, auch in der Literatur sind äusserst wenig solche Fälle verzeichnet und unter diesen keiner, in welchem in so kurzer Zeit die ganze Drüse entartete.)

An der Innenfläche des Cranium und der Aussenseite der Dura mater finden sich einige sehr flache, $\frac{1}{2}$ Zoll breite, aber kaum 1 Linie dicke Markschwamm. knoten, 2 derselben gehören nur der Glastafel an, welche an dieser Stelle rauh und löchrig ist, wälırend die entsprechende Stelle der Bura mater glatt und unverändert ist; die anderen gehen von der Dura mater aus und die entsprechende Stelle der Glastafel ist entweder ganz glatt oder nur weaig erodit. Viele an der Aussenseite der Bura mater prominirende Pachionische Granulationen zeichnen sich durch ihre Grösse $\left(\frac{1}{2}-1 \frac{1}{2}\right.$ Lin. Durchmesser $)$, weisse Farbe und Weichheit aus, auf der Schnittfläche quillt rahmiger Saft hervor, welcher unter dem Mikroskop dieselben histologischen Elemente zeigt, wie alle anderen Krebsknoten im Körper. Die so entarteten Granulationen bilden mit anderen nicht entarteten grössere Gruppen und lagern in tieferen Gruben des Cranium, welches an diesen Stellen fast perforirt ist. (Es ist dies meines Wissens der erste beobachtete Fall von Carcinom der Pacch. Granulat.)

Das Gehirn zeigt äusserlich keine Veränderung; in jeder grossen Hemisphäre finden sich 3-4 runde Krebsknoten von 2-6 hin. Durchmesser, sie sitzen theils in ler grauen Rinde, theils in der weissen Markmasse, die sie umgebende Hirnsubstanz ist unverändert. Die Zirbeldrüse ist zum Umfang einer kleinen Haselnuss angeschwollen, derb, weiss, oberflächlich glatt; auf der Schnittläche quillt rahmiger Saft hervor und die feinere Untersuchnng zeigt, dass die ganze Drüse in einen Krebsknoten umgewandelt ist, sie besteht aus Zellenmassen von genau derselben Form und Grösse, wie die in allen anderen Krebsknoten vorkommenden, und eigem zanten Fusergenüs, in weletłem hie und da auch die bekunten geschichteten Steinchen Jagern, aber äusserst sparsam. Die Crura der Zirbel sind wohl erhalten und unverändert. (Auch von dieser Entartung ist mir kein zweiter Fall bekannt. Der Befund einer totalen krebsigen Entartung der Zirbeldrïse bei vollkommener Seelenthäligkeit würde vor einem halben Jahrhundert von grosser Bedeutung für die Frage rom Sitze der Seele gewesen sein.)

Die rechte Lunge ist oberfächlich emphysematös gedunsen, übrigens in holem Grade ödematös und hyperämisch, frei von Krebs. Im tiefsten Theile der linken Pleurahöhle finden sich ungefälır $1 \frac{1}{2}$ Pfd. Wasser, von der übrigen Höhle durch feste aite Verwachsungen abgeschlossen. Die linke Lunge fühlt sich hart an, wie hepatisirt, unter der Pleura sind zahlreiche kleine, platte Krebsknoten zu sehen; 
die Bronchialdrïsen der linken Seite sind zu tauben- bis bühnereigrossen Krebsknoten entartet und bilden zusammen eine fast faustgrosse Masse, von ihnen aus geht die krebsige Entartung am Hilus der Lunge in deren Parenchym ein und erstreckt sich ziemlich 3 Zoll tief in dasselbe; die Wände der grossen Bronchialstämme und Gefässe sind rings von der Krebsmasse umgeben und die ersteren an einzelnen Stellen in der Krebsmasse untergegangen; das Lumen der Gefässe erscheint offen, das der Bronchien aher an den Stellen, wo ilıre Wände untergegangen sind, verengert oder ganz geschlossen. Ausserdem finden sich viele im Lungengewebe zerstrente, isolirte, kleinere und grössere Krebsknoten. Alle durch die Krebsmassen comprimirten Bronchien sind unterhalb sehr weit, strotzend mit rahmigem Eiter gefüllt, ihre Schleimhaut stark injicirt und aufgelockert, weich, saftig. Das Lungengewebe ist an verschiedenen Stellen im Zustande der Hyperämie, Splenisation, des ersten Grades der entzündliclsen Infiltration, Hepatisation und eitrigen Infiltration, hie und da haben sich auch kleine Abscesse gebildet. Die Schnittfläche der Lunge bictet daher einen sehr ungewöhnlichen bunten Anblíck dar und es bedarf der Hüllfe des Mikroskopes, um die entzündlichen und purulenten Herde won den krebsigen ïberall sicher unterscheiden zu können. Luftholiges Lungengewebe ist nur schr wenig chaiten.

Das Ilerz zeigt keine Veränderung, im Pericardium des rechten Herzohres findet sich ein 1 Linie dicker weisser lirebsknoten.

Von der Innenfäiche der vorderen Hälfte des linken Häftbeins prominirt cine grossc; weiche Markschwammmasse in der llöhe von 3-4 Zoll, sic schicht den Iliacus, Psoas, die grossen Gefüss- und Nervenstïmme vor sich her, ohne sie in ihrer Textur verändert zu laben; auf der Aussenfäclse prominirt dic Geschwulst ebenso hoch, bedeckt von den Glutueen. Das Carcinom geht vom Knochen selbst aus, welcher grösstentheils zerstört und beweglich ist, es erstreckt sich bis zum Boden der Pfanne, dasselbe ist an einer kleinen Stelle zerstört, das Ligamentum teres durch krebsige Infiltration zerfallen, in der Gelenkhöhle findet sich eine röthliche, rahmige Flüssigkeit; Synovialhaut und Knorpel sind obne Veränderung.

Am recliten Humeras findet sich am oberen Ende gleich unterhalb des Kopfes ein peripherisches Carcinom, dasselbe hat eine Jänge von $4-5$ Zoll und ist 1 Zoll dick, goht vom Periost aus, während die Rinde völlig normal ist. Nach Durchsägung des Knochens sieht man, dass die Markräume des Knochens in der Ausdelnung des äusseren Carcinoms mit weicher Markschwammmasse infiltrirt sind.

Die Achseldrüsen heider Seiten sind zu hülnereigrossen, ziemlich harten, auf der Schnittläche reticulären Krebslinoten entartet.

Die linke Glandula submaxillaris ist zu einem fast hühnereigrossen Krebsknoten entartet, die Lymphdräsen der linken Submaxillargegend bilden Convolute kleinerer runder, weicher Krebslinoten, während auf der rechten Seite nur einige vercinzelt entartet sind.

Zahlreiche Krelislinoten finden sich im Peritonacum und seinen Falten, sie sitzen sparsam zerstreut von 3-5 Lin. Durchmesser im P. parietale, ein 1 Zoll dickér in der Plica Dougl, ein 2 Zoll dicker im Mesenterium des Colon ascendens, sehr zalltreich 1 Lin. breit, platt im ganzen Gekröse; einzclne grosse Knoten im 
subperitonealen Zellgewebo am die Nieren. Die leber ist nomal, die Milz stawk vergrössert mit dem Habitus der Typhusmilz; das Pankreas ist in hohem Grade krebsig eotartet, die Entartung beginnt am Schwanz und nimmt nach dem Kopfe $\mathrm{zu}$; in ersterem sitzen zerstreute kleine, weiche, weisse Markschwammlnütchen zwischen den normalen Drüsenläppchen; nach der Mitte zu nehmen die Krebsknoten an Menge und Grösse zu und der Kopf besteht nur noch aus einem Conyolute grosser linoten, die in Verbindung mit den krebsig entarteten Glanl. Jymph. der Ungebung eine fanstgrosse Krebsmasse bilden; ungefälr zwei Zoll unterbalb des Pylorus ist das Duodenum mit dieser Krebsmasse verwachsen und von derselben in der Ausdehnung von fast einen Zoll durchbrochen. Der Magen ist normal, ebenso der Darmkanal, nur in der Schleimhant des Colon ascendens findet sich ein isolirter Krebsknoten von 4 Lin. Breite und 2 Lin. Höle, er prominirt und hat das Ansehen einer recht grossen marlkig infiltrirten typhösen Solifärdrüse; die mikroskopische Untersucluung zeigt nur die Krebselemente in den Knoten, doch macht es seine Form, Lage, Verhalten zur Schleimhaut wahrscheinlich, dass wir bier den seltenen Fall einer lirebsigen Entartung einer Solitärdrüsc des Dünndarms vor uns haben. Ein ganz ähnlicher Knoten findet sich in der Schleimhant der Zunge, entsprechend der Stelle einer grossen Papille der linken Seite der Zungenwurzel, ferner sind beide Tonsillen angeschwollen und mit kleinen, schr weichen Krebsknoten durchsetzt. (Diese letzten Befunde, insbesondere die beiden Knoten in der Schleimhant des Colon und der Zunge, gehören zI den grössten Seltenheiten.)

Die Mesenterialdrüsen sind normal, aber ein Theil der Lumbar- und Iliacaldriisen ist in Krebsknoten verwandelt. In der linken Niere sitzen $5-6$ Krebsknoten ron 3-5 Lin. Durchmesser, ron der Nebennicre gehen 2 Krebsknoten won $1 \frac{1}{2}$ Zoll Durchmesser aus, doch ist ihr Gewebe noch grösstentheils erhalten und die Kinoten prominiren grösstentheils nach aussen. Die reclite Nebenniere aber ist in eine Krebsmasse von 5 Zoll Breite und 3 - 4 Zoll Dicke umgewandelt, umschliesst den oberen Theil der Niere und ist mit ilhm verwachsen; im letzteren sitzt ein faustgrosser Krebsknoten, in welchem fast die Hälfte der' Niere untergegangen ist und der in das Nierenbecken als runde Geschwulst prominirt, ausserdem sitzen in dieser Niere noch mehrere kleine, discrete Krebsknoten. Die Harnwege sind frei.

Von Interesse war endich noch cine Misshildung, indem sich in der Mitte zwischen den inneren Schneidezälınen der Oberliefer ein fünfter kleiner, spitzer Schneidezahn in ciner besonderen Alveole fand, während die vier normalen Schneidezähne keine Veränderung zeigten.

Der grobe und feinere Bau der Krebsmassen war in allen Localitäten gleich, es war die Form des weichen Markschwammes mit weisser, an rahmigem Safte reicher Schnittfäche. Dic Zellen waren ansgezeichnet durch ihre Kleinheit (an diesen Zellen hätte die Pariser Schule viel Jernen können!), die Kerne hatten gerade die Grösse der Eiterzellen, waren rund, ihre Kernkörperchen klein, oft nur punktförmig, die Zellenmembran hob sich meist nur wenig vom Kern ab, die Form der Zellen war meist die runde, doch fanden sich auch ovale, eckige, geschwänzte etc. 